\title{
Coloraciones emotivas y temples anímicos en los Estudios acerca de la estructura de la conciencia de Husserl*
}

\author{
Affective Colorations and Moods in Husserl's \\ Studies on the Structure of Consciousness
}

\author{
ANTONIO ZIRIÓN QUIJANO \\ UNAM
}

Schwierig ist die volle Klarlegung solcher Intentionalitäten wie der Gemütsintentionalitäten überhaupt. (A VI 8 I/50b)

RESUMEN. El ensayo explora las nociones de "coloración" (o "resplandor", "luz" o "brillo") afectiva o emocional, así como la noción de Stimmung (temple anímico o estado de ánimo), tal como son expuestas en los manuscritos que integran el proyecto que Husserl denominó Studien zur Struktur des Bewusstseins, con una mención previa de otros dos textos que preceden a los Studien: las "Notas sobre la doctrina de la atención y el interés" de 1893 o 1894 (en Husserliana XXXVIII) y un pasaje del $\S 15$ b) de la Quinta de las Investigaciones lógicas. La revisión de los Studien destaca una primera noción de coloración como "sensación emocional" (Gefühlsempfindung), luego una segunda noción como una coloración afectiva transeúnte (o trascendente). Después de exponer las "expansiones" o "transferencias" emotivas, la revisión alcanza la noción de temple (Stimmung), la sitúa dentro del esquema de las vivencias afectivas y describe su carácter unitario, su motivación y su peculiar intencionalidad, señalando su relación con la conciencia de fondo y con las llamadas (por Husserl) "corrientes de sentimiento". Con ello, se ponen las bases para una revisión de la noción de temple o estado de ánimo, revisión que queda pendiente.

Palabras clave: Coloración (coloración afectiva); resplandor (resplandor afectivo); luz (afectiva); brillo (afectivo); Stimmung; temple; temple anímico; estado de ánimo; corriente de sentimiento.

ABSTRACT. This essay explores the notions of affective or emotional "coloration", "splendor", "light", or "shine", as well as the notion of Stimmung (mood, temper), as both of them are expounded in Husserl's project Studien zur Struktur des Bewusstseins and in two other texts considered as precedents of the Studien: the "Notes on the Doctrine of Attention and Interest" of 1893 or 1894 (in Husserliana XXXVIII and a passage in $\S 15 \mathrm{~b})$ of the Fifth of the Logical Investigations. The review of the Studien highlights a first notion

\footnotetext{
*azirionq@yahoo.com.mx ORCID iD: https://orcid.org/0000-0001-6864-7642. Este artículo está relcionado con el ensayo escrito en lengua inglesa "Colorations and Moods in the Studien zur Struktur des Bewusstseins", publicado en The New Yearbook for Phenomenology and Phenomenological Philosophy. Vol. 16 (2018), pp. 41-75.
}

Copyright: (C) 2019 CSIC. Este es un artículo de acceso abierto distribuido bajo los términos de la licencia de uso y distribución Creative Commons Reconocimiento 4.0 Internacional (CC BY 4.0). 
of coloration as "emotional sensation" (Gefühlsempfindung), then a second notion as a transcending (or transcendent) affective or emotive coloration. After the exposition of the emotive "expansions" or "transferences", it reaches the notion of a mood (Stimmung), situates it within the scheme of affective experiences, and describes their unitary character, their motivation, and their peculiar intentionality, pointing to their relation with background consciousness and with the socalled (by Husserl) "stream of sentiment". With this, there is enough ground for a revision of the notion of a mood, which is left for another essay.

Key words: Coloration (Affective Coloration); Splendor (Affective Splendor); (Affective) Light; (Affective) Shine; Stimmung; Mood; Temper; Stream of Sentiment.

\section{INTRODUCCIÓN}

Podrían darse múltiples justificaciones para estudiar conjuntamente los temas de la coloración o el resplandor de la afectividad y de los estados o temples de ánimo en los escritos de Husserl. En mi caso la motivación principal se encuentra en la aparente vinculación de estos temas con la descripción fenomenológica que he buscado del "fenómeno" que llamo "colorido de la vida". Pero no voy a tratar aquí de esta vinculación ni de las dificultades que veo ahora en la comprensión del "colorido" como una "coloración (resplandor, luz, brillo) afectiva" o como un carácter emotivo o afectivo, acaso vinculado con un temple o estado de ánimo. ${ }^{1}$ Bastará por el momento destacar la estrecha relación que las investigaciones de Husserl ponen de manifiesto entre aquellas coloraciones afectivas y estos temples anímicos.

Nos enfocamos en los manuscritos de investigación de Husserl dedicados a la emoción y la conciencia del valor que conforman la segunda parte del proyecto denominado Estudios acerca de la estructura de la conciencia. ${ }^{2}$ Los manuscritos que pertenecen a este proyecto forman sin duda un corpus unitario que merece ser considerado por sí mismo dado su alcance y su importancia -lo que ocurrirá sin duda una vez que aparezcan en Husserliana-. Con algunas excepciones, en lo que sigue me limitaré a la exploración de textos tomados de

\footnotetext{
${ }^{1}$ Algo puede encontrarse al respecto en el artículo mencionado en la nota anterior. Sobre el "colorido de la vida", véase Zirión (2003). Advierto que en lo sucesivo usaré "carácter afectivo" o "carácter de sentimiento" como traducciones de Gefühlscharakter, y "carácter emotivo" como traducción de Gemütscharakter. En general, vierto Gefühl como "sentimiento" (pero "afectivo" en las construcciones adjetivales) y Gemüt como "emoción".

${ }^{2}$ Este proyecto fue encomendado por Husserl a Ludwig Landgrebe, quien seleccionó y organizó un grupo de manuscritos escritos en su mayor parte durante los años de Gotinga (19011916). El proyecto, que estaba dividido en tres partes (Aktivität und Passivität -“Actividad y pasividad"-, Wertkonstitution, Gemüt, Wille -"Constitución de valor, emoción, voluntad"-, y Modalität und Tendenz -"Modalidad y tendencia"-), nunca se publicó en vida de Husserl. Está muy próximo a aparecer dentro de la serie Husserliana, en edición de Ullrich Melle y Thomas Vongehr. Véase Vongehr (2004) y Vongehr (2011), así como Melle (2012).
} 
la segunda parte de los Studien, pero no haré de ellos una exposición exhaustiva, y menos una interpretación comprehensiva; me concentraré, más bien, en los temas que aquí me ocupan -los cuales ya merecen una buena cantidad de interpretación-. Aunque el trabajo de Husserl acerca de estos temas tiene lugar dentro del contexto más amplio de la afectividad y la vida emocional, contexto que casi inevitablemente va de la mano de la teoría fenomenológica del valor, aquí no pondré particular atención en estos contextos, a pesar de su significación histórica y sistemática dentro de la teoría fenomenológica de la razón. No puede decidirse de antemano si los temas peculiares de las coloraciones y los temples emotivos, que son los que aquí interesan, tienen alguna incidencia en ese panorama más amplio. Así pues, todas las sugerencias o indicios -que acaso no sean escasos- que se encuentren en lo que sigue de tesis o doctrinas éticas o de teoría de la razón, quedarán aquí sin desarrollo. ${ }^{3}$

La investigación ulterior tendrá que tomar en cuenta otros textos de Husserl, publicados o inéditos, que o no menciono aquí o menciono sólo de paso. Vale la pena enfatizar que tomo los manuscritos de investigación del legado póstumo de Husserl en el espíritu que ha propuesto recientemente Elizabeth Behnke, a saber, "como momentos en una práctica de investigación en marcha que somos llamados a asumir prosiguiendo por nuestra parte el trabajo de la investigación fenomenológica concreta". ${ }^{4}$

Voy apenas a mencionar dos antecedentes de importancia relativos a esta temática en la obra de Husserl.

\section{ANTECEDENTES}

\section{Las "Notas" sobre la doctrina de la atención y el interés}

En estas "Notas", 5 escritas probablemente en 1893 o 1894, se encuentran ejemplos ilustrativos de varias posibles combinaciones de actos de los dos lados de

\footnotetext{
${ }^{3}$ En el contexto pleno tendrían que considerarse los cursos de Ética que Husserl impartió por la misma época que estos manuscritos. Véase Hua XXVIII (abrevio del modo usual las referencias a los volúmenes de la colección Husserliana; en la Bibliografía se encuentra la información completa), y también Hua XXXVII. Son útiles los trabajos de Mariano Crespo (2012) y Walton (2015), esp. el Cap. VII: "El sentimiento". En Zirión (2009) hice un primer acercamiento a algunos de los temas que aquí se tratan en relación con uno de los textos de la segunda parte de los Studien (un estudio crítico, escrito por Husserl en 1911, pero no publicado por él, de Geiger (1911a)). En lo que toca al tema de la vida afectiva en general, y en particular a los temples de ánimo, a las Stimmungen, debo mencionar los antecedentes de Quepons (2014a, 2014b, 2015a y 2015b).

${ }^{4}$ Behnke (2014), p. 295.

${ }^{5}$ Este texto ha sido publicado como Anexo II en Hua XXXVIII, pp. 159-189. De este tomo son todos los números de página que se dan entre paréntesis en esta sección.
} 
nuestra "naturaleza espiritual", el intelectual y el emotivo, y en particular la que se expresa con la metáfora de la coloración o, como aquí se dice, de la tintura o la tinción. Escuchamos con arrebato ${ }^{6}$ lo que alguien nos comunica; "nos sentimos muy diferente que en los casos en que observamos y entendemos con la emoción fría”. Nuestro estado de ánimo (Gemützustand), dice Husserl,

no es una suma de un acto del interés más el arrebato referido a él; más bien hay una fusión en la que ambos lados se penetran y determinan recíprocamente su carácter. El arrebato no es un vestido que se ponga sobre el acto de interés; también lo tiñe, pero de una manera que para su particularidad específica es indiferente. Por ello decimos que entendemos la palabra igualmente en el arrebato que fuera del mismo. El arrebato produce una variación en otra dimensión que es completamente inconmensurable con la dirección del entendimiento (164).

Lo coloreado, lo teñido por el arrebato, es ante todo el propio acto intelectual de interés, de atención en las palabras del otro. La tinción nos hace sentirnos "diferente" ante lo observado o lo escuchado. Husserl no precisa la contribución del interés en la fusión; pero hace la observación (que desarrollará en los Estudios) de que "el placer (...) se individualiza y se especifica según los contenidos y las especies de contenidos sobre los que recae. El placer ante un color es distinto que el placer ante un sonido, el placer ante este color distinto del placer ante aquél" (170). ${ }^{7} \mathrm{El}$ interés, entonces, puede no sólo fundar el placer o el arrebato, atenuarlo o aniquilarlo, sino también modificarlo cualitativamente. Esta modificación es también una coloración o tintura: "un estado intelectual nunca está acaso enteramente libre de coloraciones emocionales, y viceversa" (164). ¡Nótese el "viceversa"!

La inconmensurabilidad de las "dimensiones" del arrebato y del interés (de la emoción y del entendimiento) vuelve a plantearse al introducir la distinción entre el acto momentáneo y la disposición habitual ("hábito" o "postura"), la cual imparte a los actos aislados su "carácter", una "coloración determinada":

Si estamos en el hábito del arrebato, si la corriente de la voluptuosidad, de la ira, etc., hace estremecer nuestra alma, entonces cada vivencia anímica tiene una coloración determinada, tal como los mismos árboles se ven de otra manera con luz de sol y cielo claro que en medio de la borrasca. Si hemos adoptado una postura teórica, entonces lo que nos mueve es un interés puro en las cosas; pero es-

${ }^{6}$ Usaré uniformemente "arrebato" para traducir Affekt, incluso si en algunas ocurrencias podría no ser muy preciso con respecto a la intensidad del sentimiento. Por cierto que, salvo otra indicación, todas las traducciones de textos de Husserl que aparecen en este trabajo son mías.

${ }^{7}$ Cf. en los Studien: A VI 12 II/70. Cito los manuscritos de Husserl por su signatura en los Archivos Husserl. El número después de la diagonal es el número de página del manuscrito. 
pecíficamente los actos son los mismos, así como en el paisaje los árboles son los árboles, sólo que coloreados de otro modo, iluminados de otro modo (166).

Es notable que podamos hacer de esta coloración "objeto de nuestro interés, reflexionar sobre ella, 'colocarla frente' a nosotros, considerarla”, así sea "suspendiéndola por ello provisionalmente" (166).

Además de la consideración de los temples anímicos en relación con las disposiciones y los hábitos, también se halla en estas "Notas" la caracterización de la intencionalidad reactiva (por ejemplo de la alegría) y la exposición de su papel en el surgimiento de los temples, junto con la pérdida del objeto inicial y la coloración o iluminación por transferencia que se produce.

Cuando cierta noticia provoca un arrebato de alegría, el estado o temple anímico dura, y ocurre que "todo se nos alumbra": otras cosas despiertan alegría, pero no por ellas mismas, sino a consecuencia de la noticia alegre. A la inversa:

$\mathrm{Al}$ afligido le aparece todo en luz triste; pero los objetos que aparecen así iluminados no son los objetos de la tristeza, al menos no los primarios. El afligido sabe bien acerca de qué se aflige; su sentimiento está específicamente determinado por este objeto. No se aflige por los objetos que ahora contempla, aunque quizá esté inclinado a advertir en ellos también algo desfavorable y en general algo que sea apropiado para alimentar su tristeza. Pero este algo desfavorable es a menudo de otra determinación específica que la tristeza que lo llena (176).

Además del resplandor "prestado", Husserl menciona también los sentimientos de segundo grado, el surgimiento en un temple de motivos nuevos para prolongarse, $\mathrm{y}$, finalmente, la mezcla de sentimientos (177), antecedente de la concepción de los temples como sentimientos unitarios, y la noción de la corriente de sentimiento que fluye en una suerte de ritmo. Es igualmente interesante la consideración de la posibilidad de estados anímicos duraderos con una motivación no aparente, y la distinción en ellos entre la motivación (o la referencia a la motivación) y la intencionalidad. ${ }^{8}$

Investigaciones lógicas, $V, \S 15$ b)

Husserl ilustra en este pasaje la manera como las sensaciones de sentimiento ( $G e$ fühlsempfindungen), que carecen de intencionalidad, participan en la formación

${ }^{8}$ Es casi seguro que el investigador Nam In Lee no tuviera conocimiento de estas "Notas" cuando afirmó que el manuscrito de los Studien zur Struktur des Bewusstseins (él se refiere al "Typoskript" producido por Landgrebe) "es el primero que trata de la fenomenología del temple de ánimo" (1998, p. 104). 
de las vivencias intencionales (actos) de sentimiento (Gefühlsacte). ${ }^{9}$ Se trata de la primera referencia en su obra publicada al carácter afectivo (esplendor, coloración, brillo, etc.), y del único intento de explicación de la generación de dicho carácter. Esta explicación es notable porque contraviene (al menos en una de sus interpretaciones posibles) la tesis expresa en las mismas Investigaciones según la cual las vivencias fundadas, como las de sentimiento, no aportan nada a la materia del acto básico fundante, sino que son pura cualidad. ${ }^{10}$ El pasaje reza: ${ }^{11}$

Así, por ejemplo, la alegría por un suceso feliz es seguramente un acto. Pero este acto, que no es en efecto un mero carácter intencional, sino una vivencia concreta y eo ipso compleja, comprende en su unidad no sólo la representación del suceso alegre y el carácter de acto del agrado referido a este, sino que la representación se enlaza con una sensación de placer, que es aprehendida y localizada por un lado como excitación afectiva del sujeto psicofísico que siente y por otro lado como propiedad objetiva: el suceso aparece como bañado de un resplandor rosado ["velo rosado" en la traducción de García Morente y Gaos], el placer aparece como algo en el suceso. ${ }^{12} \mathrm{El}$ suceso de esta manera coloreado de placer, como tal, es apenas ahora el fundamento para el volverse alegremente, para el agrado, complacerse o como quiera que se llame. Igualmente, un suceso triste no es meramente representado en su contenido y nexo cósicos, en lo que le pertenece en sí y por sí como suceso, sino que aparece revestido con la coloración subjetiva ${ }^{13}$ de la tristeza. Las mismas sensaciones de desplacer que el yo empírico refiere a sí y localiza en sí (como dolor en el corazón) son referidas, en la aprehensión afectiva del suceso, ${ }^{14}$ a este mismo. Estas referencias son puramente representativas; un modo esencialmente ${ }^{15}$ nuevo de intención reside tan solo en el ser repelido hostilmente, en

${ }^{9}$ Recuérdese que en las Investigaciones lógicas un acto es genéricamente una vivencia intencional. La grafía con "c" en "Akt", "Gefühlsakt", etc., es sólo propia de la primera edición de las Logische Untersuchungen.

${ }^{10}$ Lo ha visto Serrano de Haro (1995), p. 73.

${ }^{11}$ Hua XIX/1, p. 408. Sigo el texto de la primera edición, modificando libremente la traducción de García Morente y Gaos en Husserl (1982), pp. 509-510, que fue hecha sobre la segunda. Señalo en notas aparte las divergencias entre ambas ediciones que tienen cierta importancia.

${ }^{12}$ En la segunda edición, Husserl suprimió: "el placer aparece como algo en el suceso". Seguramente Husserl prefirió evitar la ambigüedad que permitía pensar que, gracias a esta segunda apercepción, en el suceso objetivo aparecía algo de la índole del placer mismo. Pues el resplandor rosado (siguiendo con el ejemplo), por muy subjetivo que sea, no parece realmente identificable con el placer, que es vivencia.

${ }^{13}$ En la segunda edición, Husserl suprimió "subjetiva".

${ }^{14}$ En lugar de "en la aprehensión afectiva del suceso", la segunda edición dice: "en el volverse al suceso".

${ }^{15}$ El adverbio "esencialmente" fue añadido en la segunda edición. 
el desagrado activo, etc. Las sensaciones de placer y de dolor pueden perdurar, mientras que desaparecen los caracteres de acto edificados sobre ellas.

No entraré aquí en los detalles de la nada sencilla interpretación del pasaje. ${ }^{16}$ Está claro que el "resplandor rosado", o la "coloración (subjetiva) de la tristeza", se producen debido a la aprehensión intencional, a la referencia objetiva que hace el yo empírico de una sensación vivida de placer o desplacer (un sentimiento de sensación, en todo caso), hacia el objeto o el suceso captado o representado. La sensación vivida es aprehendida como una suerte de sensación exhibidora que muestra el resplandor (o la coloración de la tristeza) en el objeto o el suceso. Esta aprehensión o referencia se acompaña de otra por la cual la misma sensación es apercibida y localizada en el sujeto, en su cuerpo, como "excitación afectiva" o como "dolor en el corazón". Tenemos aquí un caso notable de aprehensión doble de una sensación, sobre la cual Husserl tenía todavía mucho que enseñarnos. ${ }^{17}$ Ya no está claro de qué índole es esa aprehensión o referencia primera que transfiere una sensación a una objetividad o que hace que la sensación funcione para exhibir en la objetividad una ("su") coloración o esplendor. Parece forzoso que esta aprehensión sea parte esencial de la misma vivencia compleja que se describe, del acto de alegría conformado por la "representación del suceso alegre y el carácter de acto del agrado referido a éste", y que sea una suerte de "efecto" del carácter de acto del agrado; es decir, que sea, como Husserl lo dice explícitamente (en el texto de la segunda edición), la "aprehensión afectiva del suceso". Sin embargo, el mismo Husserl complica la interpretación cuando dice: "Estas referencias son puramente representativas; un modo esencialmente nuevo de intención reside tan solo en el ser repelido hostilmente, en el desagrado activo, etc.". Parecería entonces que la aprehensión de la sensación de sentimiento que la objetiva como algo en el suceso tiene lugar antes de que intervenga la intencionalidad afectiva propiamente dicha. Tendríamos así un suceso meramente representado que poseería ya una cualidad por la cual puede aparecer "vibrando placenteramente". ${ }^{18}$ La intencionalidad nueva, específicamente afectiva, surgiría sólo en presencia de este suceso que es objetivamente "placentero" o "desplacentero".

Lo que aquí dejo a un lado es la discusión de la manera como propongo salir de esta extrañísima situación. ${ }^{19}$ Sólo indicaré que mi tesis estriba en reco-

${ }^{16}$ Debo remitirme aquí al artículo citado en la primera nota.

${ }^{17}$ Recuérdese la descripción de la constitución del cuerpo en Hua IV, § 36.

${ }^{18}$ Así lo expresa Serrano de Haro (1995), p. 73.

${ }^{19}$ Con el artículo citado en la primera nota, véase también Lee (1998), p. 111; Quepons (2014a), pp. 126-132; Melle (2012), pp. 56-57; Vendrell Ferran (2008), p. 201. Expresamente rechazo la lectura que hice de este pasaje en Zirión (2009), en que asumí que el calificativo de "puramente representativas" significaba algo así como "meramente dependientes de la aparición", 
nocer que Husserl describe en el pasaje dos vivencias emotivas y no sólo una: un acto inicial de valoración y la alegría o repulsión reactiva. Es decisiva aquí la ambigüedad con que Husserl usa el término "agrado" (Gefallen), la cual viene a ser otro precedente de la distinción que hará en los Studien entre acto de agrado dirigido al valor y una reacción que ya supone el primer acto como base. Hay que notar que, a pesar de la complicación creada, el pasaje apenas fue modificado en la segunda edición de la Investigación Quinta en 1913, y lo fue principalmente para retirar de él las expresiones que daban a entender que podía estar sosteniendo que en el objeto tenía lugar una vivencia o algo subjetivo. Quizá podamos decir que las dificultades de interpretación de los ejemplos paralelos expuestos en este pasaje son reflejo de las dificultades que ofrece comprender el fenómeno mismo que se investiga. Pronto se verá que lo que en esos ejemplos se ilustraba no era más que un caso posible, una posibilidad entre varias otras de combinaciones vivenciales que traen consigo coloración.

\section{LOS ESTUDIOS ACERCA DE LA ESTRUCTURA DE LA CONCIENCIA}

Articulo la exploración en diversos títulos que van presentándose en una lectura cronológica de los textos, sin revisar cada uno de ellos en forma separada. ${ }^{20}$ En relación con las cuestiones que no habían sido tratadas en sus publicaciones anteriores -las Investigaciones lógicas, sobre todo-, no hay un vocabulario técnico establecido y todos los conceptos están todavía en proceso de formación y fijación. Esto vale en primer lugar de la noción misma de temple de ánimo (Stimmung). Sólo cuando sea indispensable, recurriré a nociones que surgen en escritos posteriores de Husserl.

\section{La coloración como sensación emotiva}

La coloración afectiva o coloración de sentimiento se identifica en primer lugar con las mismas "sensaciones emotivas" (Gemütsempfindungen) que "pertenecen a las sensaciones empíricas inferiores" (los contenidos primarios de la "sensibilidad externa"). Son "coloraciones inmanentes de sensaciones sensibles" (A VI 30/220b), por ejemplo, alguna sensación de placer vivida al tener una sensación de color, entendidas ambas como vivencias no intencionales. No parece posible identificar estas sensaciones de sentimiento o emotivas (que aquí

\footnotetext{
"meramente subjetivas". Esto iría contra el lenguaje que Husserl viene utilizando en toda la Investigación $\mathrm{V}$.

${ }^{20}$ Melle (2012) ofrece una exposición ordenada de cada uno de los textos de esta segunda parte de los Studien.
} 
se llaman "coloraciones" y también, en texto posterior, "tonos de sentimiento") (A VI 12 II/69) con aquellas sensaciones de placer o de desplacer que tienen una participación esencial en el pasaje de la Investigación Quinta que revisamos. La sensación de placer que vivo al escuchar una noticia feliz no parece estar vinculada a alguna sensación sensible, a algún contenido primario; pero es una vivencia igualmente no intencional. Por su parte, la sensación de sentimiento asociada a un contenido primario es también, por supuesto, placentera o desplacentera. Pero ambas, también, sólo pueden ser aprehendidas o apercibidas intencionalmente. Una de las dos aprehensiones posibles de las sensaciones de placer en el pasaje de la Investigación Quinta da como resultado la aparición del resplandor en el suceso; pero esta aprehensión parece que es (éste era el punto medular de la discusión) sólo representativa, esto es, bien entendida, objetivante. Ahora, la apercepción empírica y unitaria de las sensaciones de sentimiento funda "un agrado o desagrado ante el objeto" (A V 12 II/27b28a), es decir, una vivencia intencional afectiva, valorativa. Se distingue entonces, con toda claridad, el sentimiento de sensación que radica "ante y en" (am und $\mathrm{im}$ ) la sensación misma, y el sentimiento ante el objeto, el agrado ( $\mathrm{Ge}$ fallen). El primero funda el segundo o es "motivo" de él. El objeto agrada, gusta, "por mor de" ese tono afectivo de la sensación; pero que la sensación tenga ese tono afectivo, esa coloración, es algo que no tiene ningún fundamento, ninguna razón. La sensación (el contenido primario) agrada o desagrada "por mor de sí misma". La sensación de sedosidad o de suavidad táctil agrada sin "porqué"; la tela sedosa o el sentimiento de suavidad contra la piel agradan (ahora como un acto intencional) sobre la base de aquel primer agrado o placer no intencional (cf. A V 12 II/69b).

¿Ocurre algo semejante en el ejemplo del "suceso feliz" en la Investigación Quinta? ¿Sabemos acerca de la "felicidad" del suceso sólo porque tenemos una sensación afectiva de placer al escuchar la noticia acerca de él? ¿No tiene este placer una razón?

\section{Coloraciones emotivas transeúntes (trascendentes)}

Esta intencionalidad del agrado (del acto emotivo en general, del cual el agrado es sólo un ejemplo conveniente), ${ }^{21}$ que Husserl llama "carácter emotivo", y que es un "modo", análogo al modo de la creencia en la percepción, es la que confiere "coloración emotiva", coloración de agrado (coloración de sentimiento), al objeto (re)presentado (sea sensible o categorial). "Si un objeto me agrada,

${ }^{21}$ En estas discusiones la palabra "acto" tiene aún el sentido que tenía en Investigaciones lógicas: vivencia intencional en general. 
si lo observo con bienestar, entonces él tiene la coloración afectiva agradable. El agrado está referido al objeto, tiene en él su 'intencionalidad"' (A VI 30/224b; A VI 12 II/30). Estas coloraciones se llaman también "interpretaciones emotivas" y son "coloraciones emotivas transeúntes" (A VI 30/220b). ${ }^{22}$ En este contexto, el uso del término "transeúnte" significa que la coloración está en el objeto (trascendente), y es lo que da lugar después a los predicados "bello", "bueno" y similares "predicados emotivos" (A VI 30/219-219b, 220a, 224b).

Respecto de esta coloración o este carácter de luz, puedo poner atención en la suscitación en mí del encanto, de la alegría, del agrado, o puedo ver el encanto como un carácter en el objeto "sin pensar que estos caracteres estén referidos a la suscitación en mí" (A I 16/10a). La luz reside en el objeto; ella lo colorea, le da un carácter, irradia de él. No es el embeleso en el sujeto lo que lanza luz sobre el objeto. La coloración es un carácter objetivo, pero es desde luego un carácter constituido, y es la conciencia la que nos habla de él: "Vivo en el encanto, entonces lo objetivo no sólo está conciente como en la conciencia de un objeto apercibido sensiblemente, o quizá incluso como un objeto de pensamiento, sino que es dado a la conciencia con un carácter de belleza radiante" (A I 16/10a). Puedo también volverme al objeto (no a su belleza radiante), o volverme "a la luz, a la belleza radiante, al carácter emotivo" (A I 16/10b).

Así, cuando el objeto es dado originariamente como objeto de un sentimiento, "no tenemos una aprehensión empírica, sino una coloración emotiva del objeto; el objeto está ahí como coloreado y como tal es intuido, percibido, cuando dirigimos a él la mirada" (A I 16/11a). Husserl deja muy claramente sentado que "el carácter de luz, lo irradiante (lo oscuro, triste), está en el objeto", aunque en formulaciones ocasionales una "coloración" parece pertenecer al sentimiento, o ser el sentimiento mismo: "un sentimiento, digamos una cierta coloración de placer" (A VI 12 II/96a).

Ahora bien, la cualidad (Beschaffenheit) del objeto que se constituye a partir del carácter de sentimiento puede ser distinta en distintos lados o superficies del objeto; en algunos de ellos puede ser positiva, bella, en otros indiferente o fea, según la unidad de sentimiento correspondiente (A VI 12 II/96a-96b). Este es precisamente uno de los defectos que Husserl encuentra en la imagen del resplandor o de la iluminación: que no responde de las diferencias que se advierten con respecto a los distintos lados del objeto: "La imagen del iluminar no es en todo respecto adecuada. (...) Los propiamente amenos son ciertos lados o momentos, pero todo el objeto está bañado de luz" (A VI 12 II/133a-133b).

${ }^{22}$ El adjetivo de Husserl, "transient", que traducimos como "transeúnte", es un antecedente del término posterior "transzendent" ("trascendente"), y parece tener esencialmente el mismo sentido. 
La coloración, que puede presentarse en la fantasía (A VI 12 II/31b) tan bien como en la percepción, no tiene relación con la creencia, pues "la creencia no "colorea" (A VI 30/220a). Tampoco colorean el deseo o el querer. La coloración la da siempre sólo la apercepción emotiva, valorativa (A VI 30/221a-221b; A VI 12 II/29a).

Ahora bien, la apercepción de valor se funda en la apercepción empírica, pero no es empírica (A VI 12 II/29a). El “carácter de luz" que brilla en el objeto "no pertenece a la apercepción objetiva, a la capa de aprehensiones de sensación. Pertenece a otra dimensión" (A I 16/10b). Husserl dirá lo mismo respecto del valor del objeto -valor que está en el origen de este carácter de luz, del resplandor-, pero valor y resplandor no son lo mismo. Aunque en algunos pasajes esta distinción no queda destacada con toda claridad, hay otros que no dejan lugar a dudas. El valor en el objeto o del objeto, o en o de alguno de sus momentos, es el fundamento del resplandor, no el resplandor mismo.

\section{Primeras expansiones y transferencias}

Tenemos hasta aquí tres distintos objetos de coloración: 1) vivencias completas (en las "Notas sobre la doctrina de la atención y el interés") en virtud de vivencias del otro "lado" de nuestra "naturaleza espiritual"; 2) sensaciones sensibles en virtud de los sentimientos sensibles que se les unen, y 3) objetos intencionales de vivencias en que domina una apercepción emotiva, sean objetos como todos o partes o superficies de los mismos. En este último caso, la alegría es suscitada por el valor del objeto, valor constituido en una valicepción (Wertnehmung): la alegría que engendra el valor de la amada presente ante mí genera a su vez una iluminación que recae sobre el objeto, sobre la amada. "Lo amable tiene 'iluminación', que tiene su fuente en la alegría. Lo valioso suscita alegría, y la alegría ilumina lo valioso" (A VI 12 II/132b). Una primera ampliación ocurre cuando la alegría se "construye" sobre una retención: la amada "ha salido del cuarto", pero yo sigo vuelto a ella (A VI 12 II/134a): las "imágenes de recuerdo", las "representaciones" u objetivaciones "vacías", son ahora la base del volverse valorando y de la alegría. La luz sigue iluminando a la amada imaginada, recordada, aunque quizá con cierto oscurecimiento o atenuación. Una primera transferencia de la luz ocurre cuando el objeto amado, o el suceso feliz, está ausente pero se habla de él: "la luz de la alegría fluye de la frase hacia lo dicho como tal y, a través de él, hacia el estado de cosas" (A VI 12 II/134a). Las palabras mismas participan del resplandor: el nombre de la amada, por ejemplo (A VI 12 II/88a); todo en la habitación que la amada ha dejado, un libro, los muebles, la habitación misma, ha "tomado atractivo y valor" por mor de su referencia a la presencia anterior de la amada. Esta ilumi- 
nación no es la misma que tendrían estas cosas si ellas fueran el objeto de mi dicha. El resplandor puede extenderse también sobre objetos que ya no tienen ninguna referencia a la amada (al objeto inicialmente valorado): miro al resto del mundo circundante y "me alegro de la luz que recae sobre todo". "iQué bello es el mundo!", puedo decirme (A VI 12 II/134b).

En estos casos, la coloración, el resplandor, es un resplandor prestado, transferido. Se trata de una diferencia esencial: o el "carácter de sentimiento", el color, está "exigido" por el contenido del objeto, es decir, por su valor, o no. Si lo está, el "carácter de sentimiento" es originario. Husserl habla incluso de la "belleza por transferencia", que no hay que confundir con la originaria (A VI 8 I/50b), y dice que la felicidad o la alegría "originarias" se convierten en un motivo de un temple de ánimo. Pues "el temple es motivado" (A VI 12 II/71a). Y una vez motivado, él mismo difunde "color y calor": "Todo toma color y calor del temple, todo placer crece y recibe un aflujo de calor que precisamente no viene del objeto de valor; lo indiferente es casi 'bello' (recibe brillo prestado)" (ibid.).

Esta misma transferencia o transmisión del resplandor hacia "todo", hacia el mundo circundante, es típica en los temples de ánimo, o quizá en ciertos temples determinados.

\section{Noción de temple anímico (Stimmung)}

La noción de temple anímico no alcanza nunca, en mi opinión, una determinación unívoca. ${ }^{23}$ En la primera alusión que hace al temple en estos textos, Husserl señala el estímulo que pueden recibir "mis pensamientos y mi temple anímico" (A VI 30/226a) de un acto de agrado como el que tengo al fumar un puro, junto con la conciencia del agrado y la combinación de las sensaciones. Se sugiere aquí claramente que algún temple preexistía al estímulo, y que con éste sólo recibió cierta determinación o modificación nueva. ${ }^{24}$ Podemos preguntar

${ }^{23}$ Conviene hacer explícitamente a un lado la acepción de la palabra alemana Stimmung como nombre de ciertos atributos o características de objetos, situaciones, paisajes, etc., en ocasiones considerados como susceptibles de ser descritos en poemas, pinturas u otras obras de arte. En los Studien Husserl no usa la palabra en esta acepción, aunque, por supuesto, no la desconocía. Véase por ejemplo Hua XXIII, pp. 476-477. De esta noción "óntica” de Stimmung se ocupa Geiger (1911b).

${ }^{24}$ En un texto escrito cuando más pronto en 1920, unos diez años después del manuscrito citado de los Studien, Husserl expresa una concepción completamente coherente con la que estamos exponiendo: "Todo sentimiento suscitado por nuevos datos de sensación encuentra su resonancia e influye en todo el medio de sentimiento que se reúne en la unidad del temple. Lo mismo es cierto de toda apercepción-de-valor despertada del modo que sea en el sujeto conciente, de toda unidad de la conciencia valorativa que, como una unidad de un sentimiento más altamente organizado, tiene una resonancia en el temple" (Hua XXXVII, p. 327). 
si el temple es más como un campo o un medio (una "facultad" incluso) que como un acto o un estado de sentimiento. Lo que puede ya decirse sobre la base de las descripciones de Husserl es que un temple determinado puede surgir a partir de actos emotivos, y que este es su modo de surgimiento más común y frecuente $-\mathrm{O}$ al menos el que ocurre con más frecuencia en las ejemplificaciones de Husserl-.

El acto de alegría pasa al estado de alegría, "un 'temple del alma' permanente" (A VI 12 II/126b). Cuando la alegría crece, "entro cada vez más en un temple" (A VI 12 II/128a). El placer o el disfrute son "fuente de un temple anímico"; de ellos emerge el bienestar, "un temple alegre que puede durar mucho" (A VI 12 II/71a). Tras una valoración (de una obra de arte, de una personalidad noble) "me invade un bienestar que puede durar ya pasada la valoración" (A VI 12 II/129b). Una alegría puede pasar "a excitación de alegría, a arrebato"..., "a un temple alegre" (A VI 12 II/130a). Los temples, en cuanto "estados", "son suscitados"; no son "tomas de posición" como lo son los actos; "la índole de la intencionalidad consiste aquí en que el valor 'que aparece' o que es conciente, es punto de irradiación de una suscitación, un suscitador para lo suscitado, para el estado del yo" (A VI 12 II/132a).

El temple suscitado puede luego resonar largo tiempo, aun cuando ya no esté yo vuelto a los valores que lo suscitaron. El encanto, la captación de algo hermoso, puede suscitar también, con el temple, un bienestar o placer corporal (un "dulce placer" o un "dolor agudo") localizado en el pecho. ${ }^{25}$ Este placer, advierte Husserl, no es la alegría misma (A VI 8 I/45b; A VI 12 II/129a-129b) y tampoco puede decirse que sea mi cuerpo (mi pecho, mi corazón) los que me dan placer o dolor. ${ }^{26}$ Los sentimientos sensuales en el cuerpo, aun si han sido suscitados por un temple alegre, no son el "sobre qué" de la alegría, no son el "objeto del disfrute" ( $c f$. A VI 12 II/71b). En cualquier caso, esta resonancia que el temple -o los sentimientos de alegría que hicieron surgir el temple- tiene en el cuerpo, puede desde luego ser comparada, o considerada en común, con el efecto (la "resonancia") que al inicio de esta sección acabamos de ver que los sentimientos sensuales o sensibles, así como cualquier acto de valor, tiene en el temple. La relación aparentemente esencial entre el

${ }^{25}$ El "pecho" o el "corazón" son de algún modo "privilegiados", pero no son las únicas partes del cuerpo que pueden estar involucradas: "Observo la pintura en el disfrute, 'me' inunda una felicidad. ' $M e$ ': a través de mi cuerpo pasa una corriente de placer, siento esta felicidad en el corazón, en el pecho, la inundación llega a los dedos de los pies" (A VI 12 II/71b).

${ }^{26}$ Husserl dice que "el enlace entre el estado de alegría, la inundación de dicha, etc., y la aprehensión del cuerpo, la aprehensión de sus miembros, es esencialmente diferente del enlace que hay entre el placer que da el platillo y la aprehensión del platillo. El platillo tiene sabor, da placer; mi pecho no 'tiene sabor' en el mismo sentido, no me da placer” (A VI 12 II/71b). 
temple y las resonancias en o desde el cuerpo es un tema que merece un estudio propio. ${ }^{27}$

Ahora bien, la expansión de la alegría -en el ejemplo favorito- significa al mismo tiempo la expansión de su coloración. Ya mientras hablo con la persona encantadora, sus palabras, su voz, sus gestos, todo tiene "sus coloraciones de sentimiento" (de $m i$ sentimiento) (A VI 8 I/45b). Una vez surgido el temple, "la alegría se transfiere (...), un temple anímico bueno hace aparecer todo en bella luz, hace verlo todo alegre" (A VI 8 I/45b; A VI 12 II/72a). A falta de tendencias en contra, ${ }^{28}$ el temple se propaga fácilmente: una alegría se liga a otra, vivo en un "ritmo de alegría"; "la alegría puede transferirse a todo lo que esté en el contexto" (A VI 12 II/72a). O también: el encanto "puede continuar, eventualmente en un temple de ánimo alegre, que es una repercusión, un sentimiento que se difunde sobre los contenidos de la conciencia, coloreando todos los objetos con su luz (...)" (A VI 8 I/50a).

\section{El esquema triple}

Los temples de ánimo ocupan, pues, el tercer sitio de la división de las vivencias intencionales afectivas, división que además indica un desarrollo concreto de sucesos anímicos. La conciencia, intencionalidad o apercepción del valor, tiene el primer puesto. Aquí el valor es "punto de partida e impulso" (A VI 8/49a). En segundo lugar está la reacción emocional, que Husserl casi siempre ejemplifica con la alegría, una alegría que puede desembocar en una "corriente de alegría suscitada" por el valor, una corriente con momentos sensibles que involucran al cuerpo (Körper), que lo inundan como un chubasco (A VI 8 $\mathrm{I} / 48 \mathrm{a})$. Esto es ya un temple, o le da paso muy fácilmente. ${ }^{29}$

Si la primera distinción parece estar bien clara, ${ }^{30}$ la segunda no lo está tanto. Un temple puede surgir directamente de la vivencia del valor, sin mediación de

${ }^{27}$ Este estudio ha sido emprendido desde cierta perspectiva en Quepons (2014b). Cf. también Johnstone (2012).

${ }^{28}$ Un ejemplo: "El temple afectivo (relacionado con cansancio del cuerpo) puede de entrada impedir que unas valoraciones estéticas produzcan mucho disfrute y evolucionen hasta el embeleso, hasta una corriente de alegría" (A VI 12 II/128b).

${ }^{29}$ Cuando Husserl hace explícito este esquema triple (por ejemplo en A VI 12 II/73a) deja en claro de nuevo que no hay distinción entre el concepto de un temple y el de un estado de ánimo. Véase Melle (2015), p. 10: "Es fundamental en sus descripciones [las descripciones de Husserl en la segunda parte de los Studien] la diferencia entre sensaciones de sentimiento, actos de sentimiento, reacciones de sentimiento, y estados de sentimiento (Gefühlszuständen)". El primer nivel es aquí desde luego el nivel de los sentimientos no intencionales. Con ellos, el esquema sería cuádruple.

${ }^{30}$ Véase A VI 8 I/50b. 
una reacción..$^{31} \mathrm{El}$ "arrebato" (Affekt) se funde o se confunde en igual medida con una alegría y con un temple. ${ }^{32}$ La realidad de la vida emocional rebasa, pues, el esquema en varios respectos. Puede hallarse unidad de motivación aun en el arrebato; en otros casos hay discordancias, temples contradictorios, "oscilaciones entre pena y placer" (A VI 12 II/91b-92a). Aun antes de que el temple surja, puede haber diferentes "actitudes" y "modos de ejecución del acto": puedo sentir alegría en presencia de un suceso, o puedo apercibir el suceso como gozoso; puedo entregarme a la alegría, o puedo refrenar la entrega, puedo sentirme libre para liberar mi energía, o sentirme inhibido, o estoy distraído, o "no tengo tiempo de alegrarme" (A VI 8 I/53b). Dentro de una "corriente de sentimiento", puede haber diferencias en la dirección al objeto o al suceso que despertó todo: la referencia nuclear a este objeto puede estar más o menos circundada por sentimientos "del derredor" que tienen nuevas referencias ( $c f$. A VI 12 II/92a y 92b). En el esquema "normal", la referencia intencional del acto valorativo original, con su "coloración rosada" sobre lo grato, se pierde como tal y se convierte en referencia de motivación, y la intencionalidad objetiva, con su "contenido" de "coloración", se disgrega en el arrebato de alegría, en el temple anímico suscitado (de enojo o de ira, de temor, de abatimiento, de aflicción o de entusiasmo), con mayor o menor unificación, hacia un entorno indefinido, sin límites o contornos precisos, pero inscrito en todo caso dentro de los horizontes de la corriente de conciencia singular del caso.

\section{Carácter unitario de los temples}

Podemos afirmar que la extensión de esa "mayor o menor unificación" es la medida de la formación de un temple. La unidad puede venir de la motivación inicial, esto es, desde el objeto suscitador "de todo el arrebato" (A VI 12 II/92a); pero los temples pueden también surgir -y esto parece ocurrir en la mayoría de los casos- de muy diversos orígenes y convertirse en una unidad de sentimiento. ${ }^{33}$ Husserl no declara que este carácter unitario del temple sea un carácter esencial, pero dada su esencial extensión temporal, cierta unidad de la cualidad intrínseca pa-

${ }^{31}$ Hay ejemplos en A VI 8/49a.

${ }^{32}$ Hay incluso alguna insinuación de que la distinción entre un arrebato y un temple tiene que ver con la intensidad, o la profundidad, del estado emotivo: "(La palabra 'arrebato' [Affekt] apunta a la excitación, a ser llevado a lo alto, a 'cimas de olas', sentimientos tormentosos u hondos valles; la palabra 'temple' [Stimmung] apunta más a un nivel equilibrado, en movilidad uniforme del sentimiento, positivo o abatido.)" (A VI 12 II/128a).

33 "Una unidad de temple anímico puede ser motivada por muy diferentes valoraciones y reacciones de valor. Diferentes corrientes de temple alegre se reúnen en la unidad de un temple alegre" (A VI 12 II/72b). 
rece realmente necesaria, al menos cuando el temple no es entendido estrictamente como un campo. Más aún, es importante notar que la "coloración" que el temple da a "todo lo que aparece" tiene también un carácter unitario, es un color unitario: un resplandor unitario de alegría o una oscura coloración de tristeza (A VI 12 II/72a). ${ }^{34}$ Ambas unidades se vinculan, esto sí, esencialmente.

\section{Motivación del temple}

Ya se ha dicho que la unidad del temple no exige una unidad de motivación. Por otro lado, hay temples claramente motivados; en otros casos, la motivación no es clara. Husserl se pregunta incluso si todo estado de ánimo (Gemütszustand) tiene su motivo: “¿No puede todo afligirme, estar todo ahí en color negro, sin ningún motivo?" (A VI 12 II/72b). En casos, puede no presentarse una motivación, o el temple puede perder la referencia determinada a su fundamento (A VI 12 II/135a). Aunque no da una respuesta definitiva, su discusión se inclina a afirmar la existencia de motivos para todos los temples, aunque esos motivos sólo puedan encontrarse "en el fondo de la conciencia" (A VI 12 II/72b). Parece haber siempre una referencia a la motivación más o menos escondida en el temple, un apuntar retrospectivamente a ella. ${ }^{35}$ No sigo aquí esta clara indicación de la posibilidad de una fundamentación fenomenológica de por lo menos algún tipo de psicoanálisis. ${ }^{36}$ Por lo demás, el origen, o la referencia al origen, puede ser incluso el único rasgo que distinga temples que no podrían distinguirse de otro modo. ${ }^{37}$

Pero este origen, la motivación del temple, o la referencia retrospectiva a esta motivación, en el grado en que se conserve, no es lo mismo que su intencionalidad. El temple que ha tendido la coloración de alegría sobre "todo", este temple, dice Husserl, "conserva ahí siempre una 'intencionalidad"” (A VI 12 II/72a); pero esta no se dirige "a lo motivante" (A VI 12 II/72b); el temple "no es un sentimiento dirigido al objeto de valor" (ibid.), al objeto valorado que fue justamente lo motivante.

\section{Intencionalidad del temple}

En el paso del acto a la reacción y luego al temple (al estado) la dirección original de la intencionalidad no se pierde, pero sí se transfiere y luego se expande

${ }^{34}$ Véase A VI 12 II/135b.

${ }^{35}$ Véase A VI 12 II/74b. "Por otro lado, la referencia puede ser clara, pero lo motivador mismo podría estar en la oscuridad" (A VI 8 I/50b).

${ }^{36}$ Puede recordarse, no obstante, el pasaje en $\S 56$ b) de Ideen II en el que Husserl se refiere explícitamente al "psicoanálisis": Hua IV, pp. 222-223.

${ }^{37}$ Véase A VI 12 II/74b. 
y se difunde. La expansión y la difusión significan la separación de la referencia al valor motivante y la intencionalidad objetiva que originalmente coincidían. ¿A qué se dirige entonces, intencionalmente, un temple anímico? La respuesta que Husserl da entre líneas es bastante clara. Un temple extiende su luz, su coloración, si no sobre "todo", sobre el mundo entero, sí sobre algún entorno más o menos amplio, más o menos indeterminado y vago -quizá más o menos discriminado, "selecto"-. El entorno puede abarcar también la vida del sujeto o trechos de ella pasados y/o futuros. Expresiones coloquiales como "el mundo entero" o "todo", al hablar acerca de la referencia objetiva de un temple, deben emplearse con cuidado. Pueden referirse sólo al entorno más inmediato, al campo perceptivo. Por otro lado, los estados de ánimo tienen en común con todas las vivencias afectivas ese rasgo peculiar de encerrar o dar lugar a una coloración, a un esplendor. La coloración es justamente un rasgo intencional ("noemático") fundado en la intencionalidad básica dirigida al valor. En el origen de la afectividad, si así puede decirse, el valor o el objeto de valor aparece en y a través de la coloración. Esta relación estructural se pierde en el temple: en él el valor fundante no es el valor del objeto que recibe su coloración. ¿Podría decirse que también detrás de la coloración que el temple "impone" se manifiesta un valor, aunque no sea el valor fundante? En estos Estudios Husserl no da pistas muy claras para responder a estas preguntas. Lo que está claro es que en el temple se mantiene la intencionalidad de la coloración, del resplandor. O, en otras palabras, lo que se mantiene es la intencionalidad que da ocasión a la coloración. Y esta referencia no es la misma que la de la valoración. Una cita es imprescindible:

Pero, ¿no ilumina la alegría, no hace aparecer a lo alegre como tal, a saber, en luz rosada? Y la tristeza, ¿no oscurece?, ¿no aparece lo triste como tal, a saber, en luz oscura, sombría? ¿No porta lo sagrado su halo, lo amado su aureola? Así que no hay remedio, tenemos que decir que también la alegría tiene su "intencionalidad", a saber, una cierta "referencia a su objeto". Pero ciertamente una referencia diferente de la de la valoración. (A VI 12 II/132a-132b)

La alegría de que se habla es un estado, un temple, como ha quedado expresamente dicho. Entonces, sin descartar ni restarle importancia a la cuestión del posible valor "detrás" de la coloración del temple, debe decirse que la intencionalidad de un temple es esa intencionalidad afectiva característica que consiste en hacer emerger, o en conferir, una coloración, un resplandor.

Puede ahora averiguarse a dónde apunta la intencionalidad de los temples, cuál es su dirección intencional, a qué "objeto" se dirigen. La regla es simple: 
“Cherchez la lumiére!”, “ßBuscad la luz (el resplandor, el brillo, la coloración)!”. Pero esto es propio de toda vivencia afectiva. Peculiar de los temples es el carácter exclusivamente transferido de esta iluminación, y la manera de su "diseminación". Naturalmente, esta regla tiene que seguirse en cada caso en el intrincamiento de la situación concreta y en la circunstancia fáctica concreta, y en juego con la conciencia de horizonte concreta. ${ }^{38}$

En los Studien, la unidad del temple llega a ser definida como la "unidad de la coloración de sentimiento, coloración que el entero acervo de conciencia, la esfera entera de lo que aparece, como tal, recibe por transferencia, la corriente general del sentimiento en la que nadamos" (A VI 12 II/73a); explícitamente dice que se trata de la "intencionalidad del estado de ánimo". Una vez más vemos que la coloración puede extenderse, o se extiende primariamente, a la misma vida de conciencia. La vida es lo coloreado en primer lugar. También es cierto que los temples, o ciertos temples, pueden comportarse como hábitos y pueden asimilar elementos que no son estrictamente afectivos. La receptividad para las incitaciones de la alegría, y la resistencia a las incitaciones o a los impulsos contrarios a esta alegría ( $c f$. A VI 8 $\mathrm{I} / 45 \mathrm{~b}$ y $50 \mathrm{a})$ son tendencias que despliegan el carácter habitual de un temple alegre, así como el hecho de que en él o a través de él todo el "espíritu" es puesto en juego (aun cuando no de manera activa), y no sólo su lado "emocional".

La coloración, pues, y su tendencia a difundirse, es, propongo, precisamente el rendimiento intencional del temple. Quisiera sugerir que un temple no se dirige a un solo objeto o estado de cosas, sino a un conjunto complejo, determinado en cada caso de una manera peculiar, singular y concreta, la cual puede entenderse-lo dije de paso- como una "selección" o un "recorte" del universo total de los correlatos objetivos del sujeto.

Por otro lado, el temple, o su carácter unitario, también determina la unidad cualitativa de la coloración. La unidad del temple como unidad de sentimiento, dice Husserl, "tiene su unidad de coloración" (A VI 8 I/50a). Ya se entiende que la variabilidad de los temples implica variabilidad de su intencionalidad, y ésta la del conjunto de los objetos sobre los cuales extiende su coloración. ${ }^{39}$

\footnotetext{
${ }^{38}$ Véase Quepons (2014a), caps. IV y V, y (2015b), pp. 101 y 102.

${ }^{39} \mathrm{Mi}$ concepción, que concede un papel central a la coloración o iluminación en la intencionalidad de los temples, podría cotejarse con otras posiciones (especialmente Lee (1998), pp. 114 y 115; Quepons (2015b), pp. 93-100; (2013), pp. 119, 131-133; (2014a), pp. 106 nota 186, 137, 139, 140, 154, 155, 177, 179; (2014b), pp. 54, 55, 56, 61, 62, 64, 65, 71, 72; (2015a), pp. 165 y 166; (2015b), pp. 94-101; Bernet (2006), p. 49; Melle (2012), p. 95) en varios respectos, y en particular con la insistencia mayor o menor de algunas de ellas a vincular la intencionalidad de los temples con la conciencia de horizonte.
} 


\section{Combinaciones y grados}

La variabilidad ocurre en diferentes dimensiones u órdenes, y aquí sólo puedo hacer una tosca enumeración. Varían el carácter habitual y la duración -que es un rasgo esencial de los temples-, ${ }^{40}$ y la peculiar duración de un hábito -hay hábitos "de corto plazo" y temples de corto plazo en cuanto hábitos- ${ }^{41}$. "Los temples pueden tener diferentes grados" (A VI 12 II/72b). Hay grados de incremento y de expansión, grados de afectación y de irradiación, grados de imposición o de resistencia a incitaciones opuestas, etc.

\section{Temple y fondos}

Para alcanzar una mejor idea de los temples anímicos, tenemos que considerar en alguna medida los fondos y/o trasfondos de la conciencia $-\mathrm{y}$ de su mundo circundante-, con su mayor o menor lejanía respecto de la conciencia actual, explícita. Husserl compara "el fondo de representación, la unidad de apercepción del fondo", en la percepción, con el "confuso fondo de sentimiento", que es sin duda, en este caso, el mismo temple anímico (A VI $8 \mathrm{I} / 50 \mathrm{a}) .{ }^{42}$ Pero la distinción es, desde luego, general: "Con sentimientos, como con todas las otras vivencias, tenemos la diferencia entre estar en primer plano y estar en el fondo, y en diferentes sentidos" (A VI 8 I/66a). No ocurre de otro modo con los estados de ánimo. "Estoy en un temple de fondo deprimido y leo estéticamente un poema de Goethe. La vivencia estética está en primer plano, esto es, yo ejecuto en ella una conciencia temática (...); el temple sombrío está en el fondo" (A VI 8 I/66b-67a). Husserl reconoce explícitamente (en A VI 12 I/269b) la posibilidad de que un temple sea vivido en primer plano (como conciencia "actual", en la terminología de Ideas I). Un temple de alegría, de ira o de entusiasmo pueden "tomar posesión de mi alma" y arrebatarme hasta el punto de no permitirme ocuparme de otra cosa. Pero esto también admite grados. Como el dolor, ${ }^{43}$ parece que los temples vividos pueden ocupar un mayor o menor campo de la atención, y en el extremo dominarla por completo. No se trata aquí, desde luego, de atención reflexiva ("observación" de los propios sentimientos) sobre el tem-

${ }^{40}$ Véase Quepons (2014a), p. 218; cf. (2014b), p. 54, y (2013), p. 133; y Bernet (2006), esp. p. 49. juntos).

${ }^{41}$ Quepons (2014b), p. 67, habla de habitualidades de corto plazo (una noción que pensamos

${ }^{42}$ En A VI 12 II/92a-92b se compara la conciencia del entorno de un objeto percibido con la manera como diferentes intencionalidades afectivas pueden circundar un arrebato.

${ }^{43}$ El tema ha sido estudiado en Serrano de Haro (2010). 
ple vivido. ${ }^{44}$ Pero la frontera con ella tampoco es clara. La reactivación de un temple al repetir sus motivaciones, de la que habla Husserl en su discusión con Geiger, consiste en cierta forma en actualizarlo ( $c f$. A VI 8 I/60a). Así, aunque un temple tenga un origen involuntario y pasivo, puede acompañar a mi conciencia actual durante cierto tiempo, o ésta puede acompañarlo a él, viviéndolo con la "preferencia" de la actualidad (en el sentido de Husserl). Pero el yo puede también, para decirlo figuradamente, hacerse cómplice de él, dejarse convencer por él, mimarlo, acariciarlo, nutrirlo... La repetición de las motivaciones puede responder a intereses distintos de los teóricos, obedecer "sugestiones" del temple mismo o de motivaciones y voliciones nuevas. Junto a las emociones de "segundo nivel" ( $c f$. A VI 12 II/131b), hay que admitir la posibilidad de temples de "segundo nivel", reacciones emocionales hacia el temple vivido que se desarrollan como un nuevo temple.

\section{Temple y corriente de sentimiento}

La posibilidad de ser vividos tanto en el fondo como en primer plano (o en los planos o fondos que haya) no concierne solamente a los temples anímicos, sino a toda vivencia emotiva (como por lo demás a todo tipo de vivencias en general, con la importante excepción de las "vivencias" de la conciencia interna del tiempo). Esta diferencia se cruza, pues, con la que hay entre vivencias emotivas temporalmente puntuales, singulares, por un lado, y vivencias que son temples, estados, cursos o corrientes de sentimiento duraderos (aunque no todas tengan que convertirse en hábitos). Si en este punto se admiten también grados, las posibilidades que brindan los diferentes cruces posibles son enormes ( $c f$. A VI 8 I/66b y 68b; A VI 12 I/267b y 268a-268b).

Aunque es curioso que en muchos de los ejemplos que se refieren a estados emotivos inconcientes o a estados emocionales que ocurren en el fondo o trasfondo, no utilice Husserl precisamente la expresión de "temple anímico" (Stimmung), sino la de "corriente(s) de sentimiento", ${ }^{45}$ creo que no tiene sentido preguntarnos en serio si ambas expresiones encierran un mismo concepto o distintos conceptos. Ambas expresiones carecen de una definición precisa y bien circunscrita. Tenemos por un lado incluso "corrientes de temples". Aunque algunos pasajes sugieren la existencia de una corriente o un ritmo de sentimiento permanente en el fondo de la vida

${ }^{44}$ Este es el tema que Husserl discute en su comentario al ensayo ya mencionado de Geiger (1911a).

${ }^{45}$ Sólo un ejemplo de suscitación de una corriente de sentimiento en la conciencia de fondo: "Sin estar vuelto a mi amigo, la conciencia de fondo de su presencia en casa suscita un sentimiento constante y determina toda la "corriente de sentimiento"” (A VI 12 II/68b). 
de conciencia -algo que no parece identificable con un temple de ánimo-, ${ }^{46}$ la capacidad o aptitud que tienen los temples de verse afectados, determinados, nutridos, por todo tipo de sentimientos de todo tipo y en todo nivel o plano de conciencia, vuelven muy dudosa la consideración de una corriente afectiva, emotiva, que no sea o que no esté por lo menos en alguna relación con el temple. Hay también casos de una clara sinonimia: "y a partir de ahí se ensancha un temple de buen humor, una corriente de sentimiento" (A VI 8 I/75a).

Pero quizá esta claridad no pueda ser la última palabra. No es fácil tomar decisiones acerca de términos que han sido tomados del lenguaje ordinario o de una tradición intelectual escasa y que no han sido sometidos a caracterizaciones o definiciones precisas. Si queremos aprovechar la investigación de Husserl en nuestra propia investigación, tenemos primero que intentar una ulterior clarificación de la noción misma de temple de ánimo. Pero tenemos que dejar esta clarificación para otra ocasión. ${ }^{47}$

\section{REFERENCIAS BIBLIOGRÁFICAS}

Behnke, Elizabeth A. (2014): "Husserl's Forschungsmanuskripte and the Open Horizon of Phenomenological Practice", Studia Phenomenologica 14 (2014), pp. 285306.

Bernet, Rudolf (2006): "Zur Phänomenologie von Trieb und Lust bei Husserl”, en Dieter Lohmar y Dirk Fonfara (eds.), Interdisziplinäre Perspektiven der Phänomenologie. Neue Felder der Kooperation: Cognitive Science, Neurowissenschaften, Psychologie, Soziologie, Politikwissenschaft und Religionwissenschaft (Dordrecht: Springer), pp. 38-53.

Crespo, Mariano (2012): El valor ético de la afectividad. Estudios de ética fenomenológica (Santiago: Ediciones Universidad Católica de Chile).

Geiger, Moritz (1911a): "Das Bewusstsein von Gefühlen”, en Alexander Pfänder (ed.), Münchener Philosophische Abhandlungen: Theodor Lipps zu seinem sechzigsten Geburtstag gewidmet von früheren Schülern (Leipzig: Barth), pp. 125-162.

Geiger, Moritz (1911b): "Zum Problem der Stimmungseinfühlung”, Zeitschrift für Ästhetik und allgemeine Kunstwissenschaft, 6, pp. 1-42.

${ }^{46}$ Husserl se refiere, por ejemplo, al "ritmo de la vida del nivel inferior con sus representaciones, juicios, sus valoraciones y esfuerzos", el cual puede "traer consigo una capa más profunda (o más elevada) de sentimiento en el ritmo sin un volverse" (A VI 12 II/135a-135b).

${ }^{47}$ En el artículo citado en la primera nota hice un primer ensayo de esta clarificación, en una sección final que en español habría llevado el título "Para una revisión de la noción de temple anímico -y un atisbo del colorido de la vida". Aquí no me queda más que remitir al lector a esa sección. 
Husserl, Edmund, Husserliana - Edmund Husserl Gesammelte Werke:

Husserliana III/1: Ideen zu einer reinen Phänomenologie und phänomenologischen Philosophie. Erstes Buch: Allgemeine Einführung in die reine Phänomenologie, nueva ed. Karl Schuhmann (Den Haag: Martinus Nijhoff, 1976).

Husserliana IV: Ideen zu einer reinen Phänomenologie und phänomenologischen Philosophie. Zweites Buch: Phänomenologische Untersuchungen zur Konstitution, ed. Marly Biemel, (Den Haag: Martinus Nijhoff, 1952).

Husserliana XIX/1: Logische Untersuchungen. Zweiter Band, Erster Teil: Untersuchungen zur Phänomenologie und Theorie der Erkenntnis, ed. Ursula Panzer (The Hague/Boston/Lancaster: Martinus Nijhoff, 1984).

Husserliana XXIII: Phantasie, Bildbewusstsein, Erinnerung. Zur Phänomenologie der anschaulichen Vergegenwärtigungen. Texte aus dem Nachlass (1898-1925), ed. Eduard Marbach (Den Haag: Martinus Nijhoff, 1980).

Husserliana XXVIII: Vorlesungen über Ethik und Wertlehre 1908-1914, ed. Ullrich Melle (Dordrecht: Kluwer Academic Publishers, 1988).

Husserliana XXXVII: Einleitung in die Ethik. Vorlesungen Sommersemester 1920/1924, ed. Henning Peucker (Dordrecht: Kluwer Academic Publishers, 2004).

Husserliana XXXVIII: Wahrnehmung und Aufmerksamkeit. Texte aus dem Nachlass (1893-1912), eds. Thomas Vongehr y Regula Giuliani (Dordrecht: Springer, 2004).

Husserl, Edmund (1982): Investigaciones lógicas, versión española de Manuel G. Morente y José Gaos (Madrid: Alianza Editorial [Alianza Universidad, 332]), Vol. 2 (1a. ed. Revista de Occidente,1929).

Husserl, Edmund (2013): Ideas relativas a una fenomenología pura y una filosofía fenomenológica. Primer libro: Introducción general a la fenomenología pura, Nueva edición y refundición integral de la traducción de José Gaos por Antonio Zirión Q. (México: UNAM/FCE).

Johnstone, Albert A. (2012): “The Deep Bodily Roots of Emotion”, Husserl Studies 28, pp. 179-200.

Lee, Nam-In (1998): “Edmund Husserl's Phenomenology of Mood”, en Natalie Depraz y Dan Zahavi (eds.), Alterity and Facticity. New Perspectives on Husserl (Dordrecht: Kluwer Academic Publishers), pp. 103-120.

Melle, Ullrich (2012): "Husserls deskriptive Erforschung der Gefühlserlebnisse”, en Roland Breeur y Ullrich Melle (eds.), Life, Subjectivity \& Art: Essays in Honor of Rudolf Bernet (The Netherlands: Springer Netherlands), pp. 51-99.

Melle, Ullrich (2015): “'Studien zur Struktur des Bewusstseins': Husserl Beitrag zu einer phänomenologische Psychologie," en M. Ubiali y M. Wehrle (eds.), Feeling and Value, Willing and Action. Essays in the Context of a phenomenological Psychology (Switzerland: Springer International), pp. 3-11. 
Quepons Ramírez, Ignacio (2013): "Nostalgia y anhelo. Contribución a su esclarecimiento fenomenológico", en Open Insight 4, pp. 117-145.

Quepons Ramírez, Ignacio (2014a): Intencionalidad de horizonte y vida afectiva. Un estudio sobre Husserl (México: Universidad Nacional Autónoma de México, tesis doctoral inédita).

Quepons Ramírez, Ignacio (2014b): "El temple de ánimo y los horizontes de la vida corporal. Esbozo de una sistematización fenomenológica", Anuario Colombiano de Fenomenología 8 (Medellín: Universidad de Antioquia, 2014), pp. 53-72.

Quepons Ramírez, Ignacio (2015a): “Apercepción de valor y tonalidad afectiva: problemas de la fenomenología husserliana de los sentimientos", Investigaciones fenomenológicas 12 (Madrid: UNED), pp. 157-183.

Quepons Ramírez, Ignacio (2015b): “Intentionality of Moods and Horizon Consciousness in Husserl's Phenomenology" en M. Ubiali y M. Wehrle (eds.), Feeling and Value, Willing and Action: Essays in the Context of a Phenomenological Psychology (Switzerland: Springer International), pp. 93-103.

Serrano de Haro, Agustín (1995): “Actos básicos y actos fundados. Exposición crítica de los primeros análisis husserlianos”, Anuario filosófico 28, pp. 61-89.

Serrano de Haro, Agustín (2010): “Atención y dolor: análisis fenomenológico”, en Agustín Serrano de Haro (ed.), Cuerpo vivido (Madrid: Ediciones Encuentro), pp. 123-161.

Vendrell Ferran, Ingrid (2008): Die Emotionen: Gefühle in der realistische Phänomenologie des Gefallens (Berlin: Akademie Verlag).

Vongehr, Thomas (2004): "Husserl über Gemüt und Gefühl in den Studien zur Struktur des Bewusstseins" en Beatrice Centi y Gianna Gigliotti (eds.) Fenomenologia della Ragion Pratica. L'etica di Edmund Husserl (Napoli: Bibliopolis), pp. 227-253.

Vongehr, Thomas (2011): "Husserls Studien über Gemüt und Wille”, en Verena Mayer, Christopher Erhard, Marisa Scherini y Uwe Meixner (eds.) Die Aktualität Husserls (Freiburg: Alber), pp. 335-360.

Walton, Roberto J. (2015): Intencionalidad y horizonticidad (Bogotá/Cali: Editorial Aula de Humanidades, Universidad de San Buenaventura).

Zirión Q., Antonio (2003): “Sobre el colorido de la vida. Ensayo de caracterización preliminar", en Acta Fenomenológica Latinoamericana I (Lima: Pontificia Universidad Católica de Perú), pp. 209-221.

Zirión Q., Antonio (2009): "El resplandor de la afectividad”, en Acta Fenomenológica Latinoamericana, III (Lima/Morelia: Círculo Latinoamericano de Fenomenología / Pontificia Universidad Católica del Perú / Universidad Michoacana de San Nicolás de Hidalgo), pp. 139-153. 